\title{
Red Quasars and Quasar Evolution: the Case of BAL QSO FIRST J155633.8+351758 ${ }^{1}$
}

\author{
Joan Najita, Arjun Dey, \& Michael Brotherton \\ KPNO/NOAO 2 , 950 N. Cherry Ave., P. O. Box 26732, Tucson, AZ 85726 \\ najita, dey, mbrother@noao.edu
}

\begin{abstract}
We present the first near-infrared spectroscopic observations of the radio-loud broad absorption line QSO FIRST J155633.8+351758. The spectrum is similar to that of a reddened QSO and shows strong emission lines of $\mathrm{H} \alpha$ and $\mathrm{H} \beta$ as well as strong Fe II emission blends near $\mathrm{H} \beta$. The redshift of the object, measured from the $\mathrm{H} \alpha$ and $\mathrm{H} \beta$ lines, is $z_{\mathrm{BLR}}=1.5008 \pm 0.0007$, slightly larger than the redshift of $z_{\text {metal }}=1.48$ estimated from the broad metal absorption features. Thus, the broad metal absorption features are blue shifted with respect to the systemic velocity. The width of the $\mathrm{H} \alpha$ emission line $\left(\mathrm{FWHM} \approx 4100 \mathrm{~km} \mathrm{~s}^{-1}\right.$ ) is typical of that observed in QSO broad-line regions, but the Balmer decrement $(\mathrm{H} \alpha / \mathrm{H} \beta \approx 5.8)$ is larger than that of most optically selected QSOs. Both the Balmer decrement and the slope of the rest-frame UV-optical continuum independently suggest a modest amount of extinction along the line of sight to the broad-line region $\left(E_{B-V} \approx 0.5\right.$ for SMC-type screen extinction at the redshift of the QSO). The implied gas column density along the line of sight is much less than is implied by the weak X-ray flux of the object, suggesting that either the broad emission and absorption line regions have a low dust-to-gas ratio, or that the rest-frame optical light encounters significantly lower mean column density lines of sight than the X-ray emission. From the rest-frame UV-optical spectrum, we are able to constrain the stellar mass content of the system $\left(<3 \times 10^{11} \mathrm{M}_{\odot}\right)$. Comparing this mass limit with the black hole mass estimated from the bolometric luminosity of the QSO, we find it possible that the ratio of the black hole to stellar mass is comparable to the Magorrian value, which would imply that the Magorrian relation is already in place at $z=1.5$. However, multiple factors favor a much larger black hole to stellar mass ratio. This would imply that if the Magorrian relation characterizes the late history of QSOs, and the situation observed for F1556+3517 is typical of the early evolutionary history of QSOs, central black hole masses develop more rapidly than bulge masses.
\end{abstract}

Subject headings: quasars: emission lines; galaxies: active; galaxies: evolution

\footnotetext{
${ }^{1}$ Based on observations at the Kitt Peak National Observatory.

${ }^{2}$ The National Optical Astronomy Observatories are operated by the Association of Universities for Research in Astronomy under Cooperative Agreement with the National Science Foundation.
} 


\section{Introduction}

Some 10-20\% of quasistellar objects (QSOs) show blue shifted, broad absorption in highionization lines (e.g., C IV and N V) in their rest-frame UV spectra (Weymann et al. 1981; Becker et al. 2000). These broad absorption line QSOs (BALQSOs) may provide valuable clues to the origin of characteristic features of QSOs (e.g., outflow phenomena, obscuring tori) and to the life history of QSOs. For example, in BALQSOs, outflow phenomena are readily apparent, which makes it easier to study their ejection dynamics for comparison with the predictions of dynamical theories (e.g., Murray \& Chiang 1995; Scoville \& Norman 1995). The underlying physics inferred from such comparisons may apply to a broader class of QSOs since several lines of evidence suggest that BALQSOs are simply normal QSOs viewed along a particular line of sight, i.e., closer to edge-on, along a line of sight that skirts an obscuring torus or wind from an accretion disk. The torus and wind may be related dynamically, e.g., if the BAL absorption arises in material ablated off the obscuring torus (e.g., Weymann et al. 1991; Voit, Weymann \& Korista 1993). The evidence in favor of this orientation-dependent unification scenario includes the fact that the emission-line properties of BALQSOs are quite similar to those of non-BALQSOs (Weymann et al. 1991), and the higher polarization (e.g., Ogle 1997; Ogle et al. 1999; Schmidt \& Hines 1999) and larger reddening (e.g., Sprayberry \& Foltz 1992; Turnshek et al. 1994) of BALQSOs compared to non-BALQSOs. In particular, spectropolarimetric studies of BALQSOs find evidence for at least two lines of sight to the QSO: a direct, edge-on line of sight that is perhaps attenuated, plus a less extincted, scattered (and hence polarized) line of sight that passes above the disk wind or torus (Hines et al. 1995; Cohen et al. 1995; Goodrich \& Miller 1995). If the incidence rate of BALQSOs among QSOs is indeed determined by orientation, then what we learn about the mechanics of ejection based on BALQSOs may be applicable to QSO outflows in general.

Following a different line of argument, BALQSOs may represent a particular evolutionary phase in the QSO lifecycle and, therefore, the fraction of QSOs that are BALQSOs may reflect the fraction of time that any given QSO spends in a BAL phase. So, for example, the red color of BALQSOs may suggest a large line-of-sight extinction and the possibility that we are viewing this object in an 'embryonic' state (e.g., Sanders et al. 1988; Voit et al. 1993). Consequently, what we learn from the study of BALQSOs may provide clues to the early stages of the life history of QSOs. As a more specfic example, in their study of red, high-z QSOs, the large Balmer decrements of Hawaii $167(z=2.36 ; \mathrm{H} \alpha / \mathrm{H} \beta=13)$ and Q0059-2735 $(z=1.59 ; \mathrm{H} \alpha / \mathrm{H} \beta=7.6)$ led Egami et al. (1996) to hypothesize that these are young QSOs forming in a shroud of massive stars and dust.

The object of the present study, FIRST J155633.8+351758 (hereinafter F1556+3517), bears

some resemblance to these red QSOs and is itself an extreme and unusual member of the BALQSO class. As summarized by Becker et al. (1997), it is a rare, iron low-ionization BALQSO discovered through optical follow up of the FIRST radio survey, and has been proposed as the first radio-loud BALQSO known (i.e., the ratio of rest-frame $5 \mathrm{Ghz}$ to $2500 \AA$ flux density is > 1000; Becker et al. 1997). F1556+3517 is also one of only a handful of QSOs to display absorption from metastable states of Fe II (Becker et al. 1997). Moreover, it is very red ( $B-K \simeq 6.6$; Hall et al. 1997), 
redder than almost all QSOs at $z \simeq 1-2$, suggesting a large column density of dust along our line of sight.

Like other BALQSOs, F1556+3517 is highly polarized in the rest-frame UV continuum $(\approx 13 \%$

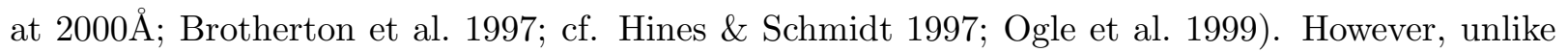
other BALQSOs, the emission lines and continuua are similarly polarized, and the BAL troughs are unpolarized (Brotherton et al. 1997). As described by Brotherton et al. (1997), the polarized component is apparently light from the QSO nucleus that is scattered into our line of sight by a scatterer located, unconventionally, outside the broad emission and absorption line regions, producing a similar level of polarization in the emission lines and the continuum. The unpolarized light in the BAL troughs may be either light from a starburst or reddened, unabsorbed QSO light viewed directly (i.e., not absorbed in the BAL region). In this paper, we use near infrared (rest-frame optical) spectroscopy of F1556+3517 to constrain the extinction toward the BAL region and to explore the stellar content of the system.

\section{Observations}

We observed F1556+3517 on U.T. 1997 May 22 and U.T. 1997 June 23 using the near-IR Cryogenic Spectrometer (CRSP; Joyce et al. 1994, Joyce 1995) at the Cassegrain focus of the 4-m Mayall Telescope of the Kitt Peak National Observatory. During the May run, the spectrograph was configured with the $300 \mathrm{l} / \mathrm{mm}$ grating (\#1) which was used in 2nd order with the $H$ filter to cover the wavelength range $\lambda \lambda 1.526-1.704 \mu \mathrm{m}$ (dispersion $\approx 7 \AA$ pixel $^{-1}$ ) and in $3 \mathrm{rd}$ order with the $J$ filter for the range $\lambda \lambda 1.1705-1.2785 \mu \mathrm{m}$ (dispersion $\approx 4.2 \AA$ pixel $^{-1}$ ). Observations of the nearby G5V star HR6538, made at approximately the same airmass and immediately following the observations of F1556+3517, were used to correct the spectrum for telluric absorption and for relative flux calibration. Our May observations were obtained through a $1^{\prime \prime}$ (2.7 pixels) slit, resulting in resolution FWHMs of $\approx 19 \AA$ and $11 \AA$ in the $H$ and $J$ bands respectively. During the June run, we used the $200 \mathrm{l} / \mathrm{mm}$ grating (\#4) which was used in 3rd order with the $I$ filter to cover $\lambda \lambda 0.894-1.103 \mu \mathrm{m}$ (dispersion $\approx 8.2 \AA$ pixel $^{-1}$ ), in 2 nd order with the $J$ filter to cover $\lambda \lambda 1.067-1.3916 \mu \mathrm{m}$ (dispersion $\approx 12.7 \AA \mathrm{pixel}^{-1}$ ), and in $2 \mathrm{nd}$ order with the $H$ filter to cover $\lambda \lambda 1.447-1.755 \mu \mathrm{m}$ (dispersion $\approx 12.1 \AA$ pixel $^{-1}$ ). The G1V star HR6064 was observed at roughly the same airmass as a telluric standard. The June observations were obtained through a 1. .'5 (4 pixels) slit, resulting in resolution FWHMs in the $I, J$ and $H$ bands of $\approx 33 \AA, 51 \AA$ and $48 \AA$

respectively. Conditions were photometric on both runs, but poor seeing and image motion during the June observations resulted in larger slit losses.

The data were dark subtracted, flat-fielded, sky subtracted and coadded using a bad-pixel mask before the spectra were extracted. Relative flux calibration for the May and June observations was performed using HD203856 and HD136754 respectively (Elias et al. 1982). The extracted spectra from the two runs were coaveraged and scaled to match the broad band photometry reported by Hall et al. (1997) using the ONIS filter transmission curves. The relative 
flux calibration of the two data sets are in excellent agreement, and the object shows no evidence for variability in the broad emission lines between May and June 1997. The data were reduced and analyzed using $I R A F$ and custom $I D L$ routines.

\section{Results}

The near-infrared spectroscopic data were combined with the optical spectrum of Brotherton et al. (1997), as shown in Figure 1. The apparent discontinuity in the continuum between the $J$ and $H$-band observations is consistent with the continuum shape of other low-ionization BALQSOs which show broad, unexplained features redward of $\mathrm{H} \beta$ in their Fe II-subtracted spectra (e.g., the MgII BALQSO 07598+6508; Boroson \& Meyers 1992). The optical spectrum is shown at the flux scaling adopted by Brotherton et al. (1997). Since the correction is small, no correction was performed for the Galactic extinction toward the object $\left(A_{V}=0.05\right)$.

The observed continuum spectral energy distribution is red and strong emission lines of $\mathrm{H} \alpha$ and $\mathrm{H} \beta$ are readily apparent. A closer look at the near-IR region of the spectrum (Figure 2) reveals that Fe II emission is present near $\mathrm{H} \beta$. In order to estimate the continuum shape in this region and to measure emission line properties, an Fe II emission template (Boroson \& Green 1992) was convolved with a gaussian with a width of $80 \AA$ (i.e., $4900 \mathrm{~km} \mathrm{~s}^{-1}$, comparable to the width of the Balmer lines), scaled, and subtracted from the observed spectrum. The central wavelengths, fluxes, and widths of the emission lines, as measured from the Fe II-subtracted spectrum, are tabulated in Table 1. The QSO redshift, as determined from the mean redshift of the $\mathrm{H} \alpha$ and $\mathrm{H} \beta$ lines, is $z_{e m}=1.5008 \pm 0.0007$, where the error represents half the difference between the redshifts implied by the two lines. Thus, the redshift is larger than that determined by Becker et al. (1997) for this object $(z=1.48)$ from broad metal absorption features in the rest-frame UV and is in closer agreement with the redshift of $z=1.497 \pm 0.001$ estimated by Brotherton et al. (1997) from a comparison of weak emission features in the rest-frame UV spectrum of F1556+3517 and a composite QSO spectrum. Given that the presence of BAL absorption makes it difficult to locate the systemic velocity of metal (absorption and emission) lines, our redshift estimate is likely to be the most robust of the three estimates. Thus, the broad metal absorption features are blue shifted with respect to the systemic velocity of the object.

The ratio of the $\mathrm{H} \alpha$ and $\mathrm{H} \beta$ line fluxes is large $\left(F_{\mathrm{H} \alpha} / F_{\mathrm{H} \beta} \approx 5.8\right)$, much above the canonical case B value of 2.87 and typical values for QSOs ( 3.5; Rudy 1984), but well within the range of observed values for QSOs $(\mathrm{H} \alpha / \mathrm{H} \beta=3-10$; Baker 1997), and less extreme than the values measured by Egami et al. (1996) for Hawaii 167 and 0059-2735 (13 and 7.6, respectively). The $\mathrm{H} \alpha$ and $\mathrm{H} \beta$ line profiles are consistent given the uncertainties associated with the Fe II subtraction. The $\mathrm{H} \beta$

\footnotetext{
${ }^{3}$ IRAF is distributed by the National Optical Astronomy Observatories, which are operated by the Association of Universities for Research in Astronomy, Inc., under cooperative agreement with the National Science Foundation.
} 
line is well fit with a single gaussian of $\approx 5200 \mathrm{~km} \mathrm{~s}^{-1} \mathrm{FWHM}$. The $\mathrm{H} \alpha$ line, measured at higher signal-to-noise ratio, is better fit as a sum of two gaussians of $2800 \mathrm{~km} \mathrm{~s}^{-1}$ and $6900 \mathrm{~km} \mathrm{~s}^{-1} \mathrm{FWHM}$, respectively, which has a combined FWHM of $4100 \mathrm{~km} \mathrm{~s}^{-1}$. The Fe II emission contributes $18 \%$ of the flux in the $J$ band but only $2 \%$ of the $H$ band flux. The Fe II and $\mathrm{H} \beta$ emission together contribute $30 \%$ of the $J$ band flux, while the $\mathrm{H} \alpha$ line contributes $69 \%$ of the $H$ band flux. The ratio of the Fe II-subtracted continua at $\mathrm{H} \alpha$ and $\mathrm{H} \beta$ for $\mathrm{F} 1556+3517\left(R_{\nu c}=1.54\right)$ is similar to the ratios reported by Egami et al. (1996) for Hawaii $167\left(R_{\nu c}=1.32\right)$ and 0059-2735 $\left(R_{\nu c}=1.67\right)$.

Neither the $\mathrm{H} \delta, \mathrm{H} \gamma$, nor strong narrow emission lines were detected. The feature near $\lambda 4340 \AA$ is much narrower $\left(830 \mathrm{~km} \mathrm{~s}^{-1}\right.$ ) than the $\mathrm{H} \alpha$ and $\mathrm{H} \beta$ lines and is probably not $\mathrm{H} \gamma$ emission associated with an AGN. The 1- $\sigma$ upper limits to the strengths of $\mathrm{H} \delta, \mathrm{H} \gamma,[\mathrm{O}$ III] $] 25007$, and [O II] $] \lambda 3727$, are $1.1 \times 10^{-16} \mathrm{erg} \mathrm{s}^{-1} \mathrm{~cm}^{-2}, 1.4 \times 10^{-16} \mathrm{erg} \mathrm{s}^{-1} \mathrm{~cm}^{-2}, 5.1 \times 10^{-17} \mathrm{erg} \mathrm{s}^{-1} \mathrm{~cm}^{-2}$, and $1.5 \times 10^{-16} \mathrm{erg} \mathrm{s}^{-1} \mathrm{~cm}^{-2}$. The non-detection of narrow emission lines is in accord with previous results for other low-ionization BALQSOs (e.g., Boroson \& Meyers 1992). We do not detect the $4000 \AA$ break $\left(F_{\lambda}[4050-4250 \AA] / F_{\lambda}[3750-3950 \AA]=1.03 \pm 0.02\right)$ in the $\mathrm{F} 1556+3517$ system (Figure 4), unlike the situation for Hawaii 167 reported by Egami et al. $\left(1996 ; F_{\lambda}[4000 \AA] / F_{\lambda}[3727 \AA] \simeq 1.21\right)$.

\section{Discussion}

\subsection{Extinction to the AGN}

The strength and width of the Balmer lines indicates that a large fraction of the rest-frame optical light arises from an AGN. The polarization results reported for the rest-frame UV (Brotherton et al. 1997) indicate that photons reach us through both direct and scattered lines-of-sight. If we assume that the AGN is the sole source of rest-frame optical photons and that it is viewed through both a direct line-of-sight and a scattered line-of-sight, both the observed continuum slope and Balmer decrement, when compared with intrinsic values, allow us to place independent contraints on the dust column density along the direct line-of-sight. Note, however, that the unpolarized absorption troughs in the rest-frame UV may indicate the contribution of an additional source of continuum emission (e.g., a starburst). If there is an additional continuum source, the Balmer lines would provide a better constraint on the extinction. Thus, the light we observe

$$
F_{\nu}^{\mathrm{obs}}=F_{d} e^{-\tau_{d}}+F_{s} e^{-\tau_{s}}+F_{\text {trough }}
$$

may have contributions from an extincted, direct line of sight $\left(F_{d} e^{-\tau_{d}}\right)$, a typically less extincted, scattered line of sight $\left(F_{s} e^{-\tau_{s}}\right)$, and a third, unscattered source of continuum photons that fills in the unpolarized trough bottoms $\left(F_{\text {trough }}\right)$.

The broad absorption and emission features imprinted on the scattered light spectrum of F1556+3517 (Brotherton et al. 1997, figure 2, bottom panel) constrain the scattering geometry. The scattered light spectrum could be produced in a situation in which an electron scattering cloud is located within the BLR and the BAL region (cf. Ogle 1997, figure 2), but, unconventionally, 
both the direct and scattered lines of sight pass through the BLR and BAL region. Alternatively, the scattered light spectrum could indicate that the scatterer is located beyond the BLR and BAL region, and BAL-absorbed light from the QSO reaches the observer through both direct and scattered paths. In the former case, the difference in the extinction between the two paths could reflect a spatial variation in the dust content of the BLR and/or BAL region. In either case, the difference in extinction could also be due to a spatial variation in the dust content of the region beyond the BLR and BAL region.

We account for either possibility, assuming that the scattered and direct components have the same intrinsic spectrum observed through different extinction paths. For simplicity, we assume that the scattering process is independent of wavelength, as in the case of electron scattering (e.g., Ogle 1997), and therefore

$$
F_{\nu}^{\mathrm{obs}}=F_{d}^{\prime}\left(e^{-\Delta \tau}+f\right)+F_{\text {trough }}
$$

where $F_{d}^{\prime}=F_{d} e^{-\tau_{s}}, f=F_{s} / F_{d}$, and $\Delta \tau=\tau_{d}-\tau_{s}$ represents the difference in extinction between the two paths.

\subsubsection{Reddened and Scattered QSO Light}

We can consider first the constraints placed by the rest-frame optical spectrum, which may be dominated by the contribution from the direct line of sight, i.e., $F_{\nu}^{\text {obs }} \simeq F_{d} e^{-\tau_{d}}$. For both Galactic (Savage \& Mathis 1979) and SMC (Bouchet et al. 1985) type dust, the observed Balmer decrement corresponds to a minimum $E(B-V) \leq 0.7$ for intrinsic decrement values of $3-5.8$. These reddenings are comparable to the reddening implied by the observed continuum slope between $\mathrm{H} \alpha$ and $\mathrm{H} \beta$. Modeling the QSO continuum as a power-law, $F_{d} \propto \nu^{-\alpha}$, and fitting the observed continuum slope between $\mathrm{H} \alpha$ and $\mathrm{H} \beta$, for intrinsic QSO spectra of $\alpha=(-1,0$, 1), we find $E(B-V) \simeq(0.75,0.46,0.14)$ which correspond to intrinsic decrements of $\simeq(3,3.5,5)$. Of these, the two higher extinction models predict a UV continuum level that is suppressed more strongly than is observed, while the lowest extinction model requires a highly unusual, very red intrinsic QSO spectrum (e.g., Elvis et al. 1994). Although these simple models provide a rough range for the extinction in the system, a more realistic model must include a scattered (and presumably less reddened) component, which is known to be present in the system (Brotherton et al. 1997).

To explore this possibility, we consider a situation in which the light at $2000 \AA$ is dominated by the scattered component, as is suggested by the high polarization of this spectral region (13\%; Brotherton et al. 1997). Figure 3 shows the contributions from the direct component $F_{\nu}^{\mathrm{obs}} e^{-\Delta \tau} /\left(e^{-\Delta \tau}+f\right)$ (dotted curve) and the scattered component $f F_{\nu}^{\mathrm{obs}} /\left(e^{-\Delta \tau}+f\right)$ (light solid curve) where $f=0.15$ and the difference in extinction between the direct and scattered lines of

sight is $E(B-V)=0.5$. In this figure $F_{\nu}^{\text {obs }}$ is the Fe II-subtracted spectrum (heavy solid curve). The scattered flux is $\approx 10$ times the direct flux at $2000 \AA$, the contributions are comparable at $4000 \AA$, and the intrinsic QSO spectrum is characterized by $\alpha \sim 0$ and a Balmer decrement of 
$\sim 4$. For comparison, we also show the continuum shape of an $\alpha=0$ spectrum that is extincted by the differential extinction of $E(B-V)=0.5$ suffered by the direct line of sight as well as an additional $\tau_{s}$ of $E(B-V)=0.1$ suffered by both lines of sight (dashed line). The non-zero value of $\tau_{s}$ was chosen to better match the shape of the UV continuum of the scattered component (light solid curve) at wavelengths $\lesssim 2900 \AA$.

The intrinsic spectral slope $\alpha=0$ and decrement of 4.3 is in good agreement with the observed correlation between Balmer decrements and optical slopes in radio selected quasar samples. From optical spectroscopy of a complete sample of low-frequency selected radio quasars (the Molonglo quasar sample), Baker (1997) measured Balmer decrements of $3.7-10$ and found that larger Balmer decrements are strongly correlated with steeper optical slopes, $F_{\nu} \propto \nu^{-\alpha}$ over the range $\alpha=0-2.5$. This correlation was interpreted as evidence that all quasars have similar intrinsic optical slopes $(\alpha \simeq 0)$ and Balmer decrements $(\approx 3.7$ with a spread \pm 0.5$)$ and that the range of observed values is primarily due to varying extinction along the line of sight to the AGN. Considering the two extinction estimates given above (extincted direct component only; extincted direct and scattered components) as limiting cases, it appears that F1556+3517 is low to moderately reddened, $E(B-V)=0.15-0.6$, with the actual value depending on the magnitude of the scattered contribution. Note that for a bluer intrinsic QSO continuum $(\alpha<0)$, the total reddening to the QSO would be larger than estimated here.

\subsubsection{Possible Starlight Contribution}

The third, unscattered component $F_{\text {trough }}$ has been ignored in the above discussion. This is appropriate if $F_{\text {trough }}$ has a flat spectral shape $\left(f_{\nu} \propto \nu^{0}\right)$, as in the case of a young stellar population, or if the residual trough intensity is the result of partial covering of a (flat) QSO spectrum. Given the low flux density in the UV troughs (10\% of the flux near C III] $\lambda 1909$; Brotherton et al. 1997), the contribution to the total light spectrum at longer wavelengths would be negligible in this case. However, this would not be appropriate if $F_{\text {trough }}$ rises rapidly in the red, as might be the case for an older or reddened stellar population. Brotherton et al. (1997) suggested that the emission in the unpolarized trough bottoms in the rest-frame UV spectrum of F1556+3517 is due to a stellar component, e.g., as seen in Markarian 231 (Smith et al. 1995), a red quasar with a known starburst population. The rest-frame UV and optical spectra allow us to test this hypothesis.

The four unpolarized low-ionization troughs located at rest-frame wavelengths $1860 \AA$ to $2800 \AA$ (Figure $₫$ ) and our limit on the $4000 \AA$ break constrain the possible starlight contribution to the spectrum. For example, the spectral shape defined by the four trough bottoms is fairly well fit by an unreddened 1 Gyr old population from the 1996 version of the Bruzual \& Charlot models (single burst, Salpeter IMF from $0.1-125 \mathrm{M}_{\odot}$, solar metallicity; Bruzual \& Charlot 1993). However, it overpredicts the $4000 \AA$ break and contributes $\sim 70 \%$ of the continuum at $\mathrm{H} \alpha$ which implies that the QSO rest-frame $\mathrm{H} \alpha$ equivalent width is improbably large $(\sim 1500 \AA)$. 
This old population is, therefore, unlikely to account for a significant fraction of the residual intensity in the troughs. To reduce the $4000 \AA$ break strength of the composite spectrum to within $1-\sigma$ of the measured value requires that the 1 Gyr old stellar population contribute $<15 \%$ of the flux at $4000 \AA$ and $\lesssim 20 \%$ of the flux in the trough (Figure 4 , bottom curve). Assuming $H_{0}=80 \mathrm{~km} \mathrm{~s}^{-1} \mathrm{Mpc}^{-1}$ and $q_{0}=0.5$, the stellar mass of this population is $<3.3 \times 10^{11} \mathrm{M}_{\odot}$.

For comparison, we can also consider the limit placed on a possible starlight contribution from a younger stellar population. If we model the trough light as arising entirely from a 50 Myr old population, we find that the spectral shape defined by the trough bottoms requires an extinction of $E(B-V)=0.45$ for the SMC extinction law (Figure 1 , middle curve). Since the intrinsic spectral energy distribution of a 50 Myr old population is flatter than that of a 1 Gyr old population, scaling the reddened model spectrum to match the flux density in the troughs implies a reasonable $\mathrm{H} \alpha$ equivalent width. Although the younger population can contribute a greater fraction of the light at $\sim 2000 \AA$ compared to the 1 Gyr population, the upper limit on the mass is lower in this case $\left(1.8 \times 10^{11} \mathrm{M}_{\odot}\right)$ due to the much smaller mass-to-light ratio of the younger population. Given these considerations, we conclude that if starlight contributes some fraction of the trough light, the flux contribution is small and has a negligible impact on the extinction estimates presented in the previous sections. We return in section 4.5 to a discussion of the possible stellar mass of the F1556+3517 system and its implications for the evolutionary history of QSOs.

\subsubsection{Comparison with Previous Extinction Estimates}

There have been a number of previous estimates of the reddening toward F1556+3517, each employing a variety of techniques and assumptions. Our analysis is more robust than previous estimates in that it uses the continuum slope and Balmer decrement as independent constraints and includes the contribution from a scattering component. Nevertheless, our extinction estimate is, perhaps fortuitously, in surprising general agreement with previous estimates. For example, from near-infrared photometry of F1556+3517, Hall et al. (1997) used the $B-K$ color of the object $(B-K=6.57)$ to infer the extinction in the system. They argued that since an intrinsic $B-K=3$ is typical of QSOs at the redshift of $\mathrm{F} 1556+3517(z=1.5), E(B-V)=0.63$ assuming SMC-type extinction. In retrospect, this analysis suffers from the assumption that F1556+3517 is a typical QSO when in fact strong BAL absorption depresses the flux shortward of $2800 \AA$ (rest frame), creating the artificial impression of a larger extinction. For example, the dereddened spectrum of $\mathrm{F} 1556+3517$ (section $4.1 .2 ; E(B-V)=0.5$ ) has a $B-R$ color of 2.25 , whereas the mean QSO spectrum from the FIRST Bright Quasar Survey (Brotherton et al. 2000), when placed at the redshift of $\mathrm{F} 1556+3517$, has $B-R=0.55$.

In another study, Clavel (1998) used 4-16 $\mu \mathrm{m}$ narrow-band images of F1556+3517 obtained with ISOCAM to estimate the extinction. Comparing the measured infrared fluxes to published optical fluxes yielded an optical-IR spectral index of $\alpha=-2$. Assuming an intrinsic slope of $\alpha=-0.5$ as likely and assuming a Galactic extinction law, Clavel determined $A_{V}=1.6$. Whether 
observations extending over such a large range of wavelengths could be adequately modeled by screen extinction is a concern, since the flux at thermal infrared wavelengths may be affected by the reemission of extincted, reprocessed radiation, and the flux contribution at optical wavelengths is now known to have a significant contribution from scattered light.

Brotherton et al. (1997) estimated a higher extinction for F1556+3517 from the rest-frame UV spectrum $(E(B-V)=0.8)$, due in part to their assumption of a bluer intrinsic QSO continuum slope $(\alpha=-0.3)$ than is assumed here and the assumption that there are true continuum points in the spectrum at $\sim 1900 \AA$. Since the location of the true continuum is difficult to determine from the rest-frame UV spectrum, which is contaminated by strong BAL absorption, the constraints placed by the present study are more robust. We confirm that in a model in which the flux at $2000 \AA$ is dominated by the scattering component, the extinction could be as high as $E(B-V)=0.8$ if the intrinsic continuum slope is $\alpha \simeq-0.3$. In this case, the scattered and direct components are comparable at $4000 \AA$, the direct component contributes $\sim 80 \%$ of the flux at $\mathrm{H} \alpha$, and the intrinsic decrement is 3.4 .

\subsection{The Radio Loudness of F1556+3517}

BALQSOs in general are radio quiet objects: the ratio of their radio to optical continuum emission, generally parametrized by $\log R^{*} \equiv \log \left(F_{5 \mathrm{GHz}} / F_{2500}\right)$ (where $F_{5 \mathrm{GHz}}$ and $F_{2500}$ are the $5 \mathrm{GHz}$ and $2500 \AA$ rest-frame $k$-corrected flux densities), is small, with typical values of $\log R^{*}<1.0$ (Stocke et al. 1992). In comparison, Becker et al. (1997) report that F1556+3517 is unusually radio loud: calculating $\log R$ directly from the optical blue magnitude estimated from the POSS-I plates, Becker et al. (1997) found that $\mathrm{F} 1556+3517$ has $\log R \approx 3.18$. Given our present estimate of the extinction to the AGN, we can reexamine the issue of radio loudness of F1556+3517 and compare it to other BAL and non-BAL QSOs.

We calculated $\log R^{*}$ after correcting the optical magnitude for both reddening and redshift. The observed flux density as measured from the spectrum at $2500(1+z) \AA$ is $5.6 \times 10^{-28} \mathrm{erg} \mathrm{s}^{-1} \mathrm{~cm}^{-2} \mathrm{~Hz}^{-1}$. Correcting for extinction using an SMC-like extinction curve an $E(B-V)=0.5$ results in $\log R^{*} \approx 0.9$. The main difference between our current estimate of $\log R^{*}$ and that presented by Becker et al. (1997) is the large extinction correction: for our estimated $E(B-V)=0.5$, the corresponding correction in the $B$ band (rest frame $1760 \AA$ ) for an SMC extinction curve is $A_{1760} \approx 5.4 \mathrm{mag}$. The extinction corrected value of $\log R^{*}$ derived here places F1556+3517 at the upper end of the $\log R^{*}$ distribution of known BAL QSOs, at the borderline between radio loud and radio quiet objects, and roughly near the peak of the distribution for optically selected QSOs (e.g., Stocke et al. 1992; see also Brotherton et al. 1998; Becker et al. 2000) 


\subsection{Comparison to Hawaii 167 and 0059-2735}

The observational situation for F1556+3517 is superficially similar to that of Hawaii 167 and 0059-2735. As noted in section 3, the ratio of the continua at $\mathrm{H} \alpha$ and $\mathrm{H} \beta$ is similar for all three objects, indicating modest reddening, but the Balmer decrement of Hawaii 167 is much larger than the observed decrements of both 0059-2735 and F1556+3517, indicating substantial reddening. This discrepancy led Egami et al. (1994) to conclude that, at least in the case of Hawaii 167, the continuum shape is not a reliable indicator of the extinction to the AGN. For Hawaii 167, the extinction implied by the Balmer decrement was large enough to extinguish the rest-frame UV continuum of the AGN. As a result, the authors concluded that the UV continuum originated elsewhere, most probably in a starburst, which the UV spectrum (Cowie et al. 1994) resembles. In the case of F1556+3517, the UV spectrum does not resemble that of a starburst, and the more modest Balmer decrement leaves open the possibility that both the QSO continuum slope and the Balmer decrement probe the same, low to moderate, dust column density. In this interpretation, the extreme (observed) $B-K$ color of F1556+3517, compared to the less extreme color of Hawaii 167 , is due to the combined absence of stellar UV continuum light and the suppression of the UV continuum of the AGN by strong BAL absorption, rather than due to a significant column density of intervening dust.

\subsection{Relation Between Gas and Dust Column Densities}

It is interesting to compare the derived dust absorption column density with estimates for the column densities in BALs and the typical X-ray absorption column densities for BALQSOs. BALQSOs have been found to be weaker X-ray sources than non-BALQSOs with otherwise similar properties (Green \& Mathur 1996; Gallagher et al. 1999; Brinkmann et al. 1999), a result which is attributed, a least in part, to absorption of the X-ray flux by a substantial intervening gas column density $\left(N_{H}>10^{22} \mathrm{~cm}^{-2}\right.$ to $\left.5 \times 10^{23} \mathrm{~cm}^{-2}\right)$. Similarly large column densities are also implied in analyses of BAL features. For example, Hamann (1998) has shown that the detection of broad P V absorption in the BALQSO PG1254+047 and the strengths of the BALs in that system are consistent with solar abundance for the BALs if many of the lines are severely saturated and the total hydrogen column density is high $\left(N_{H}>10^{22} \mathrm{~cm}^{-2}\right)$. Such large column densities are estimated to provide sufficient optical depth in a large enough number of lines to radiatively drive the BAL outflow.

Evidence for a large intervening gas column density in F1556+3517 is available from a serendipitous observation of F1556+3517 with the ROSAT PSPC; F1556+3517 is not detected in an $8 \mathrm{ksec}$ exposure. If we estimate the intrinsic X-ray flux of F1556+3517 using the radio-X-ray luminosity correlation of Siebert et al. (1996) and assuming an X-ray photon index of 1.7 (cf., the radio-loud QSO B2 $1225+31$ at $\mathrm{z}=2.2$, Buehler et al. 1995), the ROSAT non-detection implies an

intrinsic $N_{\mathrm{H}} \geq 3 \times 10^{23} \mathrm{~cm}^{-2}$. In comparison, the dust absorption column density averaged along 
lines of sight to the rest-frame UV/optical continuum emitting region of F1556+3517 is $A_{V} \simeq 1.5$, which corresponds to a much lower gas column density of $N_{H} \simeq 3 \times 10^{21} \mathrm{~cm}^{-2}$, given the standard gas-to-dust ratio for the Galaxy, or $N_{H} \simeq 3 \times 10^{22} \mathrm{~cm}^{-2}$ with the gas-to-dust ratio for the SMC. If this measurement probes the equivalent line-of-sight as the X-ray measurements, then, our measured dust column density could be either intermixed with the X-ray/BAL absorbing gas or located further along the line-of-sight to the observer, i.e., outside the BAL region. In either case, the much lower dust absorption column densities that we measure may indicate that the $\mathrm{X}$-ray/BAL absorbing gas is quite dust-poor, with a gas-to-dust ratio lower even than that of the SMC.

The absence of significant dust in the broad line region (BLR) is consistent with the results of Laor \& Draine (1993) who have argued that the BLR is too close to the active nucleus for much dust to survive in the BLR. Indeed, as argued by Netzer \& Laor (1993), the radial extent of the BLR may be defined by the dust sublimation radius. If the BAL region is located just beyond the BLR, dust may survive within it. Scoville \& Norman (1995) have proposed that radiation pressure on dust supplied by circumstellar mass loss from evolved stars residing in a nuclear star cluster is responsible for the ejection of BAL outflows. In their analysis, they assume that the BAL gas is metal rich, and therefore dust-rich, with a Galactic dust-to-gas ratio. If the BAL gas column density in F1556+3517 is at least that measured for PG1254+047, we find that the BAL region has a much lower dust-to-gas ratio, $<1 / 3$ the Galactic value. Since the rest-frame UV continuum of F1556+3517 is more strongly suppressed by BAL absorption than is PG1254+047 (Steidel \& Sargent 1991), the gas absorption column of F1556+3517 is likely to be larger than that of PG1254+047 and the dust-to-gas ratio even lower than estimated above. Moreover, the possibility that the dust column we measure is not associated with the BAL also suggests that the actual dust-to-gas ratio is actually much less than the Galactic value. If most BAL regions are this dust poor, it may prove difficult to drive BAL outflows using radiation pressure on dust grains. Resonance-line driven winds (e.g., Murray \& Chiang 1995) would then be the more likely possibility.

As a caveat to the above discussion, we note that it is likely that the optical continuum and Balmer line emitting region is intrinsically much larger than the X-ray emitting region and, therefore, probes a wider range of lines-of-sight compared to observations made at X-ray wavelengths. In this case, the lower column density may result from an average over predominantly low column density lines-of-sight to the optical continuum emission region compared to higher column density lines-of-sight to the X-ray emitting region. In this case, determining the appropriate correction factor that accounts for the different lines of sight would be necessary before we can accurately compare the gas and dust column densities measured at different wavelengths. 


\subsection{Stellar Content and Evolutionary State of F1556+3517}

Magorrian et al. (1998) have shown that the black hole masses of nearby galaxies are remarkably correlated with their estimated bulge masses over 4 orders of magnitude, with $M_{\mathrm{BH}} / M_{\text {bulge }} \simeq 0.005 ;$ more recent estimates suggest a smaller proportionality constant $M_{\mathrm{BH}} / M_{\text {bulge }} \simeq 0.0017$ (Gebhardt et al. 2000). If this implies a direct relation between the growth rates of central black holes and stellar bulges, this argues that accretion on parsec size scales is somehow related to accretion on kiloparsec size scales. Silk \& Rees (1998) argue that since collapsing objects tend to form cuspy density profiles (e.g., Navarro \& Steinmetz 2000), the early formation of a massive central black hole is greatly favored over the early formation of a large number of stars distributed on galactic size scales. They also argue that a wind from the QSO that forms, if dynamically significant, i.e., possessing adequate force to reverse accretion onto the black hole, will tend to produce a correlation between black hole mass and galactic mass that is much like the observed correlation. Expanding on this hypothesis, Fabian (1999) argues more explicitly that the stellar spheroid and central black hole grow together by the coordinated accretion of gas on kiloparsec and sub-parsec size scales. The presence of cold gas in the vicinity of the black hole is responsible for the heavy obscuration that is believed to characterize the early evolutionary history of QSOs. In Fabian's picture, the powerful wind from the QSO is eventually able to sweep away the obscuration, revealing the QSO and terminating the growth of both the black hole and the stellar component of the galaxy.

Since F1556+3517 is one such dust-enshrouded QSO with a powerful outflow that may be viewed in an early evolutionary state, it is of interest to explore whether and how F1556+3517 fits into this picture. As described in section 4.1.3, we estimated an upper limit to the stellar mass of the system by assuming that a maximal fraction of the light observed in the unpolarized UV troughs is due to stars. We found that if the stellar population is of intermediate age (1 Gyr), it can contribute no more than $3.3 \times 10^{11} \mathrm{M}_{\odot}$. If the stellar population is young (50 Myr), the maximal stellar mass is $1.8 \times 10^{11} \mathrm{M}_{\odot}$. Since the $1^{\prime \prime}$ and $1^{\prime \prime} .5$ slit widths used in our observations correspond to linear dimensions of $5.5 \mathrm{kpc}$ and $8.2 \mathrm{kpc}\left(H_{0}=80 \mathrm{~km} \mathrm{~s}^{-1} \mathrm{Mpc}^{-1}\right.$ and $\left.q_{0}=0.5\right)$, observations are likely to account for the entire bulge population in the system.

We can estimate the black hole mass of the QSO from the dereddened direct component of the rest-frame $V$-band continuum flux. Using the mean QSO SED from Elvis et al. (1994), which implies that $L_{\mathrm{bol}} / L_{V}=13.2$, we obtain $L_{\mathrm{bol}}=1.8 \times 10^{13} \mathrm{~L}_{\odot}$. Assuming that the black hole radiates at a fraction $\eta$ of the Eddington luminosity, the implied black hole mass is $M_{\mathrm{BH}}=5.7 \times 10^{8} / \eta \mathrm{M}_{\odot}$. Thus, the ratio of the black hole and stellar masses is $0.0017 / \eta$. If $\eta=1$, the ratio is the same as that given by the Magorrian relation. If $\eta$ is instead a more likely value $(\eta<0.1$; Osterbrock 1989; Nulsen \& Fabian 2000), then the black hole mass is much larger than would be implied by the stellar mass given the Magorrian relation.

As described by Laor (1998; see also Laor [2000] and references therein), the $\mathrm{H} \beta$ linewidth can be used to obtain an estimate of the black hole mass and $\eta$ based on the general assumptions 
that velocities in the broad line region are governed by gravity (i.e., $v^{2} \simeq G M_{\mathrm{BH}} / R_{\mathrm{BLR}}$ ) and that the size of the BLR is set by the bolometric luminosity of the AGN. Laor (1998) has adopted a relation of the form $R_{\mathrm{BLR}}=0.086 L_{46}^{1 / 2} \mathrm{pc}$ (where $L_{46}=L / 10^{46} \mathrm{erg} \mathrm{s}^{-1}$ and $L$ is the bolometric luminosity), based on the reverberation mapping results of Kaspi et al. (1996) and other theoretical considerations. Taken together, these two assumptions imply a relation between the black hole mass, characteristic BLR velocity, and AGN luminosity of $M_{\mathrm{BH}}=0.18 \times 10^{9} \mathrm{M}_{\odot} v_{3000}^{2} L_{46}^{1 / 2}$, and, therefore, $\eta=0.44 v_{3000}^{-2} L_{46}^{1 / 2}$.

Given the relative lack of specificity in the physics underlying these relations, one would expect that although the relations might describe general correlations between AGN properties, they are unlikely to predict black hole masses accurate to better than an order of magnitude. Surprisingly, if $v$ is taken to be the FWHM of the $\mathrm{H} \beta$ line and black hole masses are predicted for a sample of PG quasars with measured bulge luminosities, the relation between bulge luminosity and black hole mass overlies the Magorrian relation (Laor 1998), a result which has been taken to imply that the scaling relation is accurate to a factor of $2-3$. It is important to bear in mind, however, that only a direct measurement of $M_{\mathrm{BH}}$ (and therefore $\eta$ ) in these systems would confirm the quantitative accuracy of such scaling relations. Therefore, we adopt a more cautious approach and regard the black hole mass predicted by such scaling relations as a speculative, but interesting possibility.

Using the above scaling relation and the measured $\mathrm{H} \beta$ width for $\mathrm{F} 1556+3517$, we find $\eta=0.40$ and $M_{\mathrm{BH}}=1.4 \times 10^{9} \mathrm{M}_{\odot}$. If $\eta=0.40$ and the stellar mass is at its maximal allowed value $\left(3.3 \times 10^{11} \mathrm{M}_{\odot}\right)$, the ratio of $M_{\mathrm{BH}} / M_{\text {bulge }}=0.004$ puts $\mathrm{F} 1556+3517$ close to the mean Magorrian relation, a result similar to that found by Laor (1998) for the PG quasar sample. In this case, the higher redshift of F1556+3517, compared to the PG quasar sample, implies that the Magorrian relation is already in place at $z=1.5$. Moreover, at the maximal stellar mass the galaxy would already have formed most of its stars. So if F1556+3517 eventually evolved to fall on the Magorrian relation, the evolution to the final black hole mass was already nearly complete at $z=1.5$.

The relatively large value of $\eta$ thus derived for $\mathrm{F} 1556+3517$ is consistent with some of its other spectral properties. The strong Fe II and weak [O III] emission of F1556+3517 place it in a group of objects that share extreme values of the Boroson and Green 'eigenvector 1' (Boroson and Green 1992). This group, which includes Narrow Line Seyfert 1s, low ionization BAL QSOs, and QSOs with weak soft X-ray emission (Brandt, Laor \& Wills 2000), share traits that were postulated by Boroson and Green (1992) to imply large accretion rates. Recent estimates by Laor (2000) based on the scaling relation described above confirm that these strong FeII QSOs have large values of $\eta$. The reason behind the high accretion luminosities (presumably driven by a high accretion rate) in these AGN is unknown: various authors have speculated that it may indicate that these AGN are young, based on the tendency for these sources to have strong associated absorption, infrared excesses, and (in the case of the nearest examples) show evidence of recent star burst activity (e.g., Mathur 2000; Becker et al. 2000, and references therein). 
However, other considerations favor a higher $M_{\mathrm{BH}} / M_{\text {bulge }}$. For example, more recent results reported by Kaspi et al. (2000) favor a steeper $R_{\mathrm{BLR}}(L)$ relation: $R_{\mathrm{BLR}}=0.17 L_{46}^{0.7} \mathrm{pc}$ (for $\left.H_{0}=80 \mathrm{~km} \mathrm{~s}^{-1} \mathrm{Mpc}^{-1} q_{0}=0.5\right)$. With this relation, $M_{\mathrm{BH}}=0.34 \times 10^{9} \mathrm{M}_{\odot} v_{3000}^{2} L_{46}^{0.7}$, and

$\eta=0.23 v_{3000}^{-2} L_{46}^{0.3}$. Therefore, in the case of F1556+3517, $\eta=0.14$ and $M_{\mathrm{BH}}=4.1 \times 10^{9} \mathrm{M}_{\odot}$. With this lower value of $\eta$ and a maximal stellar mass, $M_{\mathrm{BH}} / M_{\text {bulge }}=0.012$, significantly above the Magorrian relation.

Note also that the black hole mass is estimated conservatively. The $L / L_{\text {Edd }}$ of the active nuclei of local Seyferts are found to be as small as $\eta=10^{-4}$ (E. Agol, personal communication). In addition, a maximal scattered fraction of the F1556+3517 spectrum, as determined in section 4.1.1, has been excluded in the luminosity estimate. We have assumed that the black hole radiates isotropically, a conservative assumption given the probable edge-on orientation of the system. The extinction correction we have applied $\left(A_{V}=1.55\right)$ is modest, and unlikely to be much smaller since intrinsic QSO continua are likely to be bluer rather than redder than we have assumed.

In contrast, the stellar mass is likely to be overestimated. We have assumed that a maximal fraction of the trough light is starlight due to a 1 Gyr population, whereas a fraction could be unpolarized light from the active nucleus, either due to partial covering of the nucleus or multiply scattered nuclear emission. If we have overestimated the age of the stellar population, as is likely to be the case, the true stellar mass is even smaller. We have also assumed a Salpeter IMF, which has a more massive contribution from low mass stars than is measured for the stellar field population in the solar neighborhood. Thus, it appears likely that $M_{\mathrm{BH}} / M_{\text {bulge }}$ is larger than estimated above. Consequently, if F1556+3517 eventually evolved to fall on the Magorrian relation, then, as speculated by Silk and Rees (1998), the black hole mass developed more rapidly than the bulge mass, a massive black hole already having been in place at $z=1.5$. Whether this evolutionary history is anomalous or the norm among QSOs is an issue of considerable interest for the future.

\section{Conclusions}

Our near-IR spectrum of the unusual BALQSO F1556+3517, combined with the existing optical spectrum, places new constraints on the dust and stellar content of the system. These results suggest interesting implications for the origin of QSO outflows and the accretion history of QSOs.

Both the Balmer decrement and the rest-frame UV-optical continuum slope of F1556+3517 provide evidence for modest extinction in the system. By modelling the rest-frame UV-optical spectrum as a combination of extincted direct and scattered light components, we find that the differential extinction between the two lines of sight is $E(B-V) \approx 0.5$. The total extinction to the active nucleus may be higher if the intrinsic spectrum of the QSO is bluer than we have assumed (i.e., if $\alpha<0 ; F_{\nu} \propto \nu^{-\alpha}$ ). Since the extinction is modest, the very red $B-K$ color of 
F1556+3517 is due to the suppression of the rest-frame UV continuum by strong BAL absorption rather than due to a large column density of dust along the line of sight. For a typical Galactic dust-to-gas ratio, the implied gas column density along the line of sight to the broad line region is much less than has been inferred for F1556+3517 on the basis of its weak X-ray flux. This suggests that either the broad emission and absorption line regions have a low dust-to-gas ratio or that the rest-frame optical light encounters significantly lower mean column density lines of sight than the X-ray emission. If our results are due to a low dust-to-gas ratio, it would appear difficult to drive BAL outflows with radiation pressure on dust grains, and resonant line driven winds are a more attractive possibility.

In contrast to the result obtained by Egami et al. (1996) for the red quasar Hawaii 167, the $4000 \AA$ break is not detected in the system, implying that if the system has a significant stellar population, the population is young ( $<1 \mathrm{Gyr})$. Assuming the maximal stellar contribution allowed by the spectrum, and using the rest-frame $V$-band continuum to estimate the black hole mass, we find that if $L / L_{\mathrm{Edd}}$ is large $(\sim 1)$, the ratio $M_{\mathrm{BH}} / M_{\text {bulge }}$ is comparable to that of the Magorrian relation. In this case, the Magorrian relation is already in place at $z=1.5$. However, multiple factors favor a much larger $M_{\mathrm{BH}} / M_{\text {bulge }}$ ratio. In this case, if the Magorrian relation characterizes the late history of QSOs, and the situation observed for F1556+3517 is typical of the early evolutionary history of QSOs, this result implies that central black hole masses develop more rapidly than bulge masses.

We are very grateful to the staff of KPNO, particularly Dick Joyce and Brett Huggard, who made these observations possible, and Paul Ho who assisted with the observations. We thank Paul Martini and Pat Hall for providing us with their $K$-band image of F1556+3517 in advance of publication. We also thank Eric Agol for communicating his results on the $L / L$ edd of Seyfert galaxies in advance of publication. We are also grateful to Ed Moran and Sally Laurent-Muehleisen for assistance with the ROSAT data on F1556+3517; to Gary Bower, Todd Boroson, Richard Green, and especially Ari Laor for their comments on the manuscript; and to the anonymous referee whose thoughtful comments improved the presentation of the paper. Finally, we thank Sperello di Serego Alighieri and the staff of the Osservatorio di Arcetri for their kind and generous hospitality in Florence, where an early draft of this paper was written. 


\section{References}

Baker, J. C. 1997, MNRAS, 286, 23

Baker, A.C., Carswell, R.F., Bailey, J.A., Espey, B.R., Smith, M.G. \& Ward, M.J. 1994, MNRAS, 270,575

Becker, R.H., White, R.L., Gregg, M.D., Brotherton, M.S., Laurent-Muehleisen, S.A., Arav, N. 2000, ApJ, 538, 72

Becker, R. H., Gregg, M. D., Hook, I. M., McMahon, R. G., White, R. L. \& Helfand, D. J. 1997, ApJ, 479, L93

Boroson, T. A. \& Green, R. F. 1992, ApJS, 80, 109

Boroson, T. A. \& Meyers, K. A. 1992, ApJ, 397, 442

Bouchet, P., Lequeux J., Maurice, E., Prévot, L., \& Prévot-burnichon, M. L. 1985, AA, 149, 330

Brandt, W. N., Laor, A. \& Wills, B. J. 2000, ApJ, 528, 637

Brinkmann, W., Wang, T., Matsuoka, M. \& Yuan, W. 1999, A\&A, 345, 43

Brotherton, M. S., Tran, H. D., van Breugel, W., Dey, A. \& Antonucci, R. 1997, ApJ, 487, L113

Brotherton, M.S., van Breugel, W., Smith, R.J., Boyle, B.J., Shanks, T., Croom, S.M., \& Miller, L. 1998, ApJ, 505, L7

Brotherton, M. S., Tran, H. D., Becker, R.H., Gregg, M.D., Laurent-Muehleisen, S.A., \& White, R.L. 2000, ApJ, submitted

Bruzual A., G. \& Charlot, S. 1993, ApJ, 405, 538

Buehler, P., Courvoisier, T. J.-L., Staubert, R., Brunner, H. \& Lamer, G. 1995, A\&A, 295, 309

Clavel, J. 1998, A\&A, 331, 853

Cohen, M. H., Ogle, P. M., Tran, H. D., Vermeulen, R. C., Miller, J. S., Goodrich, R. W. \& Martel, A. R. 1995, ApJ, 448, L77

Cowie, L. L., Songaila, A., Hu, E. M., Egami, E., Huang, J. S., Pickles, A. J., Ridgway, S. E., Wainscoat, R. J., \& Weymann, R. J. 1994, ApJ, 432, L83

Egami, E., Iwamuro, F., Maihara, T., Oya, S. \& Cowie, L. L. 1996, AJ, 112, 73

Elias, J.H., Frogel, J.A., Matthews, K., \& Neugebauer, G. 1982, AJ, 87, 1029

Elvis, M. et al. 1994, ApJS, 95, 1

Fabian, A.C. 1999, MNRAS, 308, L39

Gallagher, S. C., Brandt, W.N., Sambruna, R.M., Mathur, S. \& Yamasaki, N. 1999, ApJ, 519, 549

Gebhardt, K. et al. 2000, astro-ph/0007123, ApJ, in press

Goodrich, R.W., \& Miller, J.S. 1995, ApJ, 448, L73

Green, P.J., \& Mathur, S. 1996, ApJ, 462, 637

Hall, P. B., Martini, P., DePoy, D. L. \& Gatley, I. 1997, ApJ, 484, L17

Hamann, F. 1998, ApJ, 500, 798

Hazard, C., McMahon, R. G., Webb, J. K., \& Morton, D. C. 1987, ApJ, 323, 263 
Hines, D. C., Schmidt, G. D., Smith, P. S., Cutri, R. M. \& Low, F. J. 1995, ApJ, 450, L1

Hines, D. H. \& Schmidt, G. D. 1997, in Mass Ejection from Active Galactic Nuclei, ed. N. Arav, I. Shlosman, \& R. J. Weymann (San Francisco: ASP), 59

Joyce, R.R. 1995, "Cryogenic Spectrometer Users Manual", (Tucson:NOAO)

Joyce, R.R., Fowler, A.M., \& Heim, G.B. 1994, Proc. SPIE, 2198, 725.

Kaspi, S., Smith, P.S., Maoz, D., Netzer, H., \& Jannuzi, B.T., 1996, ApJ, 471, L75

Kaspi, S., Smith, P.S., Netzer, H., Maoz, D., Jannuzi, B.T., \& Giveon, U. 2000, ApJ, 533, 631

Laor, A. \& Draine, B. T. 1993, ApJ, 402, 441

Laor, A., Jannuzi, B. T., Green, R. F. \& Boroson, T. A. 1997, ApJ, in press.

Laor, A. 1998, ApJ, 505, L83

Laor, A. 2000, astro-ph/0005144, to appear in New Astronomy Reviews

Magorrian, J., Tremaine, S., Richstone, D., Bender, R., Bower, G., Dressler, A., Faber, S.M., Gebhardt, K., Green, R., Grillmair, C., Kormendy, J., \& Lauer, T. 1998, AJ, 115, 2285

Mathur, S. 2000, MNRAS, 314, L17

Murray, N., \& Chiang, J. 1995, ApJ, 454, L105

Navarro, J.F., \& Steinmetz, M. 2000, ApJ, 528, 607

Netzer, H. \& Laor, A. 1993, ApJ, 404, L51

Nulsen, P.E.J., \& Fabian, A.C. 2000, MNRAS, 311, 346

Ogle, P. M. 1997, in Mass Ejection from Active Galactic Nuclei, ed. N. Arav, I. Shlosman, \& R. J. Weymann (San Francisco: ASP), 78

Ogle, P. M., Cohen, M. H., Miller, J. S., Tran, H. D., Goodrich, R. W. \& Martel, A. R. 1999, ApJS, 125, 1

Osterbrock, D.E. 1989, Astrophysics of Gaseous Nebulae and Active Galactic Nuclei, (Mill Valley: University Science Books), p. 342

Rudy, R.J. 1984, ApJ, 284, 33

Sanders, D.B., Soifer, B.T., Elias, J.H., Neugebauer, G., \& Matthews, K. 1988, ApJ, 328, L35

Savage, B. D. \& Mathis, J. S. 1979, ARAA, 17, 73

Schmidt, G. D. \& Hines, D. C. 1999, ApJ, 512, 125

Scoville, N., \& Norman, C. 1995, ApJ, 451, 510

Siebert, J., Brinkmann, W., Morganti, R., Tadhunter, C. N., Danziger, I. J., Fosbury, R. A. E. \& di Serego Alighieri, S. 1996, MNRAS, 279, 1331

Silk, J., \& Rees, M.J. 1998, A\&A, 331, L1

Smith, P. S., Schmidt, G. D., Allen, R. G. \& Angel, J. R. P. 1995, ApJ, 444, 146

Sprayberry, D. \& Foltz, C. B. 1992, ApJ, 390, 39

Steidel, C.C., \& Sargent, W.L.W. 1991, ApJ, 382, 433

Turnshek, D. A., et al. 1994, ApJ, 428, 93 
Turnshek, D. A., Monier, E. M., Sirola, C. J. \& Espey, B. R. 1997, ApJ, 476, 40

Voit, G. M., Weymann, R. J. \& Korista, K. T. 1993, ApJ, 413, 95

Weymann, R. J., Morris, S.L., Foltz, C.B., \& Hewett, P.C. 1991, ApJ, 373, 23

Weymann, R. J., Carswell, R. F., \& Smith, M. G. 1981, ARA\&A, 19, 41 


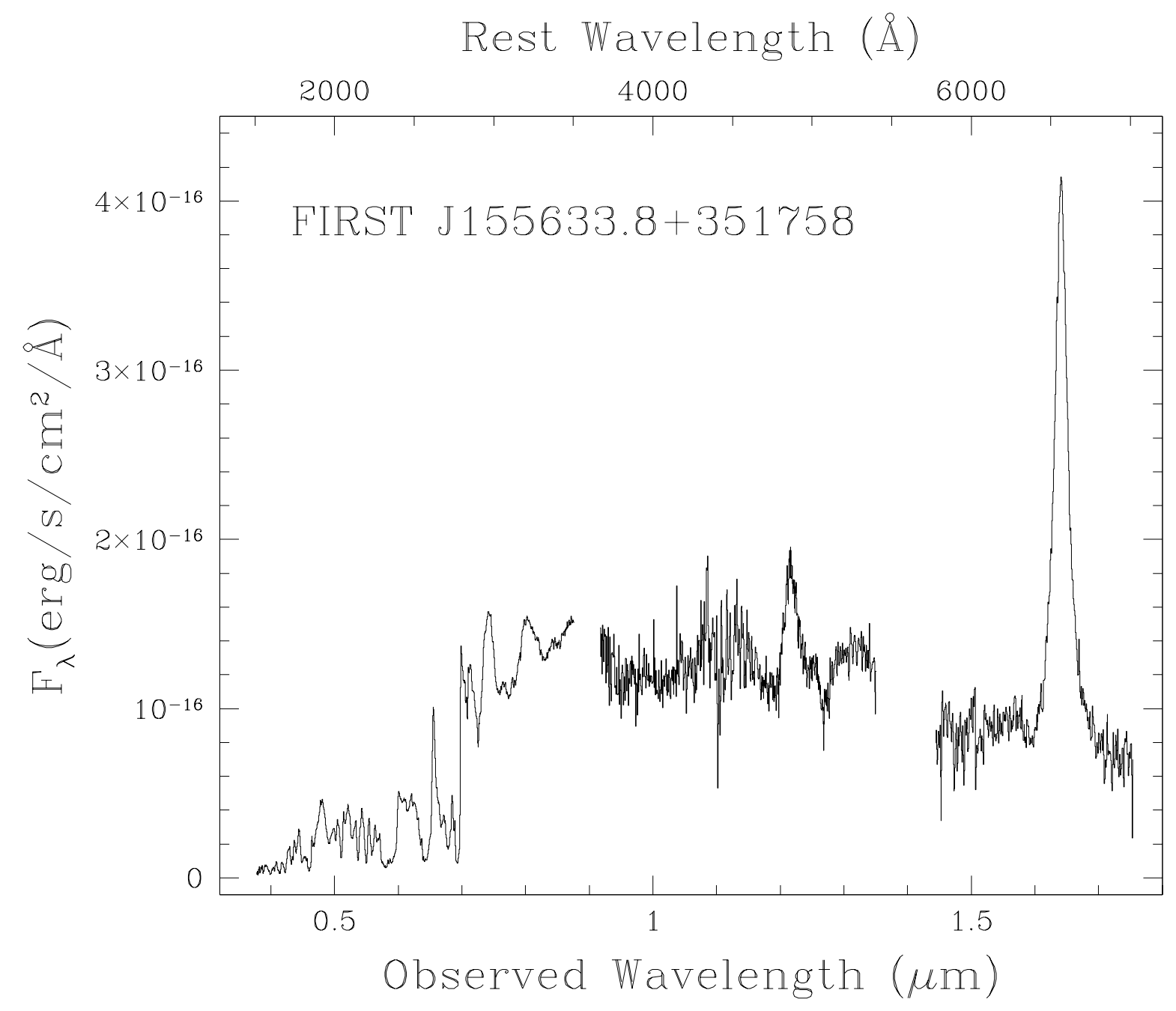

Fig. 1. - The optical and near-infrared spectrum of F1556+3517 constructed by combining the total light Keck spectrum of Brotherton et al. (1997) and the KPNO 4-m data presented in this paper. 


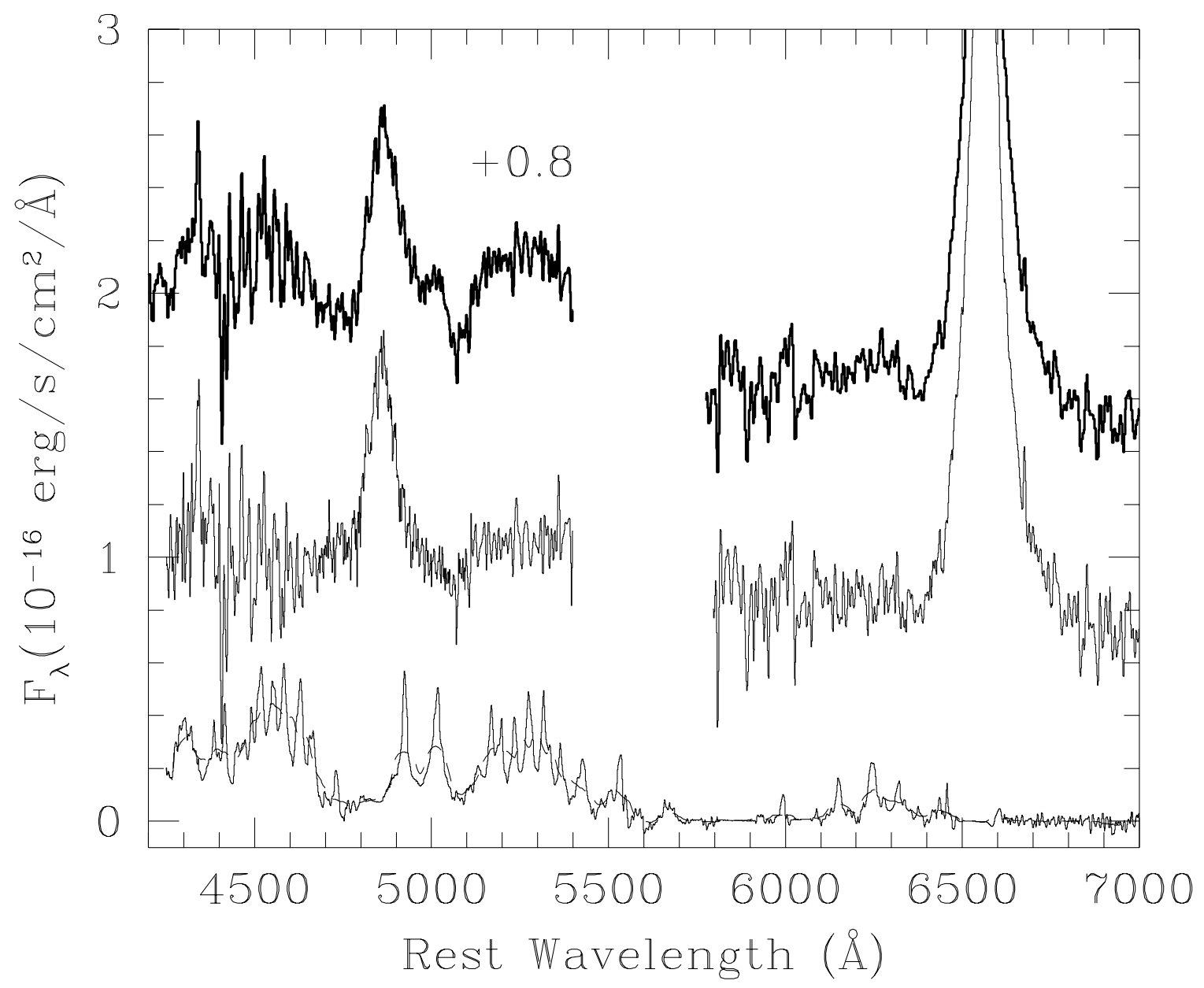

Fig. 2.- The middle spectrum shows the F1556+3517 spectrum after subtraction of the smoothed FeII emission template of Boroson \& Green (1992). The original spectrum is shown for comparison as the upper solid line (offset by $0.8 \times 10^{-16} \mathrm{erg} / \mathrm{s} / \mathrm{cm}^{2} / \AA$ ). The FeII emission template (bottom solid line) was smoothed by a gaussian of $4900 \mathrm{~km} \mathrm{~s}^{-1}$ width (bottom dashed line). 


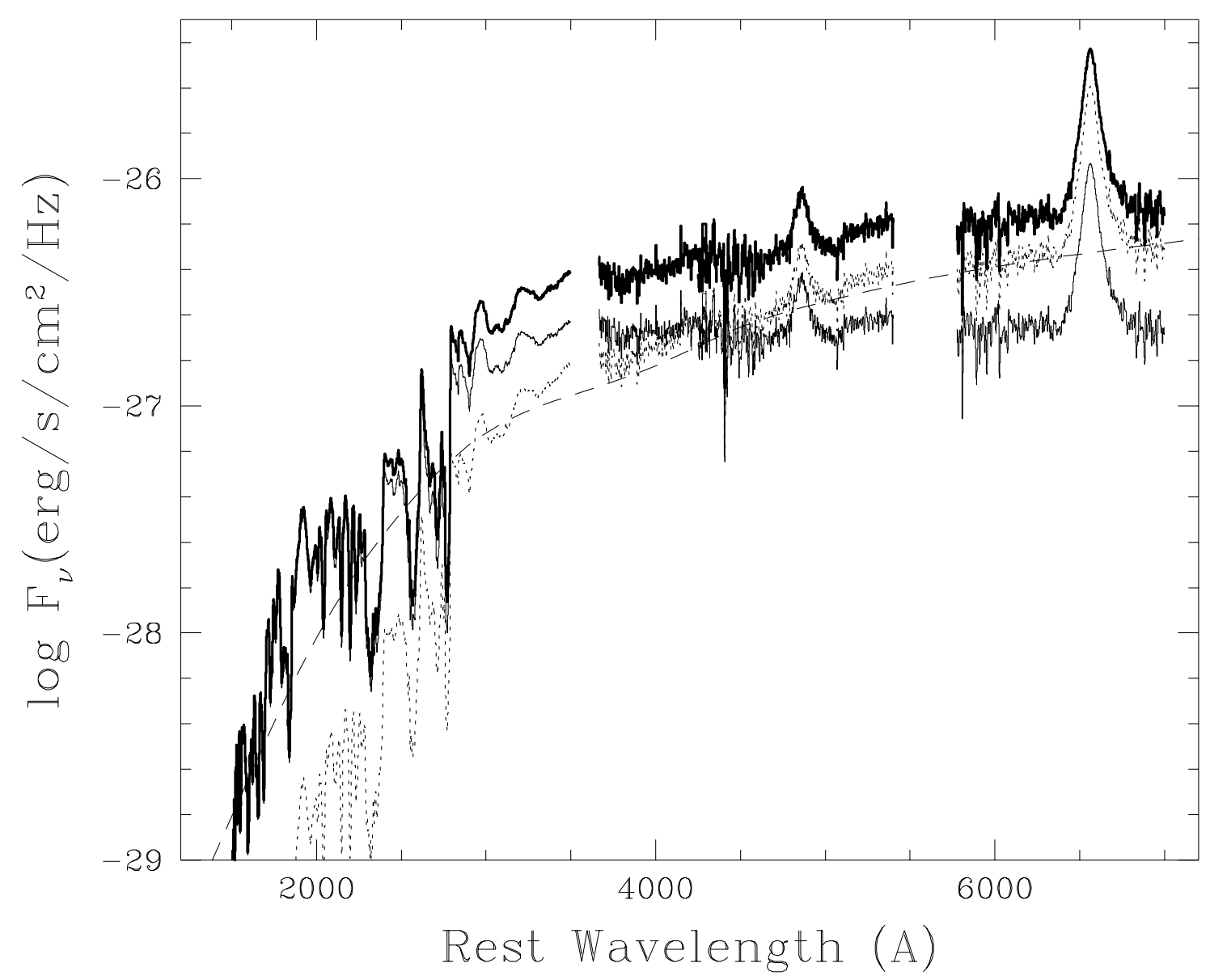

Fig. 3. - The Fe II-subtracted OUV spectrum of 1556+3517 (heavy solid line) decomposed into a scattered contribution that dominates the flux at $2000 \AA$ (light solid line) and a direct contribution that experiences a differential extinction of $E(B-V)=0.5$ (dotted line). The intrinsic QSO continuum is flat, $F_{\nu} \approx$ constant as indicated by the scattered component. The direct component is well-fit by a $F_{\nu} \propto \lambda^{0}$ continuum that is reddened by a total extinction of $E(B-V)=0.6$ (dashed line). The additional $E(B-V)=0.1$ is needed to better approximate the downturn of the continuum in the rest frame UV. 


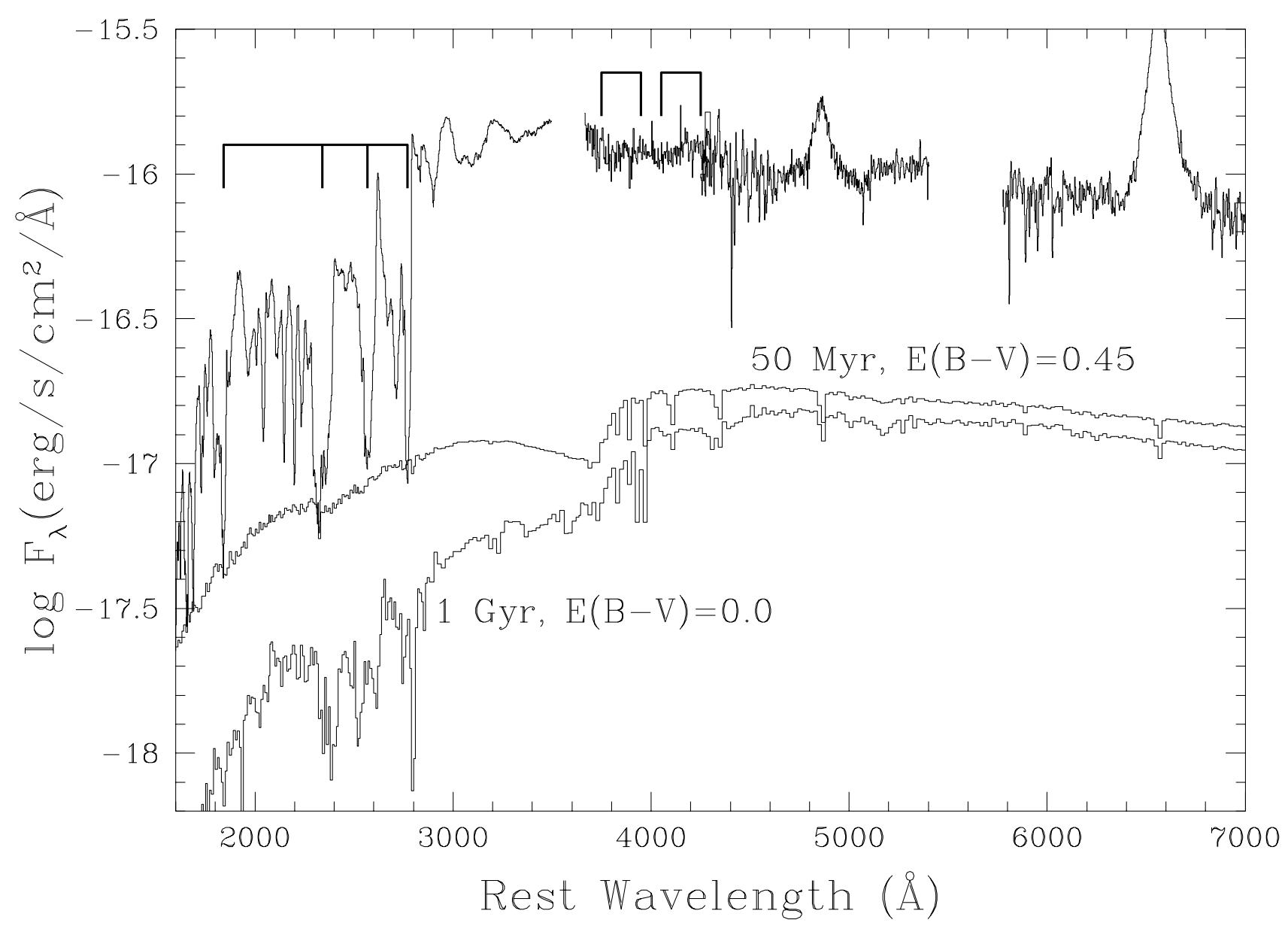

Fig. 4. - The spectral shape defined by the four trough bottoms at $\lambda<3000 \AA$ (left horizontal bar) in the rest frame UV spectrum of $\mathrm{F} 1556+3517$ (top spectrum) is well fit by a 50 Myr old stellar population extincted by $E(B-V)=0.45$ (middle curve). An older, unreddened 1 Gyr population has a similar spectral shape in the rest frame UV (bottom curve), but because of its stronger $4000 \AA$ break (as defined by the wavelength regions indicated) can contribute no more than $\sim 20 \%$ of the trough light. 
Table 1. Spectral Features

\begin{tabular}{|c|c|c|c|c|c|}
\hline Feature & $\lambda_{\text {rest }}(\AA)$ & $\lambda_{\text {obs }}(\AA)$ & $F_{\text {obs }}^{\dagger}$ & $\mathrm{FWHM}_{\mathrm{obs}}\left(\mathrm{km} \mathrm{s}^{-1}\right)$ & $W_{\lambda}^{\text {rest }}(\AA)$ \\
\hline $\mathrm{H} \alpha^{\ddagger}$ & 6562.79 & 16417.08 & 92.2 & 4112 & 441 \\
\hline H $\alpha-$ narrow $^{\ddagger}$ & & 16414.08 & 26.5 & 2815 & 127 \\
\hline $\mathrm{H} \alpha$-broad b $^{\ddagger}$ & & 16420.07 & 65.8 & 6851 & 314 \\
\hline $\mathrm{H} \beta^{\ddagger}$ & 4861.33 & 12154.17 & 16.0 & 5183 & 63 \\
\hline $\mathrm{H} \gamma$ & 4340.47 & - & $0 \pm 0.11$ & - & - \\
\hline $\mathrm{H} \delta$ & 4101.74 & - & $0 \pm 0.17$ & - & - \\
\hline [O III] & 5007 & - & $0 \pm 0.03$ & - & - \\
\hline$[\mathrm{O} \mathrm{II}]$ & 3727 & - & $0 \pm 0.17$ & - & - \\
\hline Fe II $(J$-band $)$ & $4400-5400$ & & 23.0 & & \\
\hline Fe II $(H$-band $)$ & 5800-7000 & & 2.43 & & \\
\hline
\end{tabular}

${ }^{\dagger}$ All fluxes are in units of $10^{-15} \mathrm{erg} \mathrm{s}^{-1} \mathrm{~cm}^{-2}$.

${ }^{\ddagger}$ Properties measured from Fe II-subtracted spectrum. 